\title{
Molecular Dynamics Simulation of Aggregates in the Dodecane/span80 System and Their Behaviour in an Electric Field
}

\author{
Madhusoodanan Mannoor, Sangmo Kang, and Yong Kweon Suh \\ Department of Mechanical Engineering, Dong-A University, 840 Hadan-dong, Saha-gu, Busan 604-714, Republic of Korea \\ Correspondence should be addressed to Yong Kweon Suh; yksuh@dau.ac.kr
}

Received 14 August 2015; Revised 15 October 2015; Accepted 1 November 2015

Academic Editor: Victor V. Moshchalkov

Copyright (C) 2015 Madhusoodanan Mannoor et al. This is an open access article distributed under the Creative Commons Attribution License, which permits unrestricted use, distribution, and reproduction in any medium, provided the original work is properly cited.

\begin{abstract}
Molecular dynamics simulation of self-assembly of surfactant span80 molecules to form reverse micelles in nonpolar liquid dodecane is carried out. Simulations are performed using a united atom model for dodecane and a hybrid model for span 80 molecules. Various physical characteristics of reverse micelle are measured, and the same are compared with available experimental results. Presence of charge carriers in the form of solvated ions in the core of reverse micelles is confirmed by the simulation. Movement of reverse micelles under the effect of uniform external electric field is also discussed.
\end{abstract}

\section{Introduction}

Electrohydrodynamics of dielectric liquid is a multiphysics problem involving fluid mechanics, electrochemistry, surface chemistry, and electrostatics. It is an area of growing interest to researchers, since it is associated with various technologies such as electric power industries, EHD pump, cooling of high heat flux electronic equipment, charge injection atomizers, electrophoretic image displays, and petroleum processing. Since free ions are scarcely found in nonpolar liquid, they are characterized by low electrical conductivity. However, the presence of impurities could be a source of free ions when the dielectric liquid is subjected to an electric field [1]. The characteristic length at which ions remain solvated, called Bjerrum length, is quite high for dielectric liquids compared to that of water, which would imply that free cations and anions generated due to ion dissociation can come together in a recombination process to form neutral ion pairs $[2,3]$.

It has been observed in various studies that addition of surfactant molecules increases free ion concentration [4]. This enhancement in electrical conductivity is attributed to the formation of reverse micelles by the mechanism of self-assembly of surfactant molecules around the aggregation of water molecules in the dielectric liquid [2].
In reverse micelles, the hydrophilic head portion of surfactant molecules gets attached to the water core (which is formed by the aggregation of water molecules in the dielectric liquid) and the hydrophobic tail portion gets aligned away from the water core [2]. Impurity ions can reside inside the water core, electrostatically shielded by the surfactant molecules around, thereby preventing recombination with counter ions. Reverse micelles with electric charges in the core serve as ions in nonpolar liquids [1-5].

It is also known that the conductivity of dielectric liquid depends on the concentration of surfactant [3-10]. A linear relationship has been observed between conductivity and concentration of ionizable surfactant, whereas it is nonlinear in the case of nonionizable surfactants [5].

If the diameter of the reverse micelles is smaller than the Bjerrum length, recombination of counter ions would still take place, forming neutral ion pairs. So, at any point in time, only a small fraction of the total number or reverse micelle will be charged. Accepted mechanisms of charge generation are dissociation $\left(\mathrm{AB} \Leftrightarrow \mathrm{A}^{+}+\mathrm{B}^{-}\right)$and disproportionation $\left(2 \mathrm{~A} \Leftrightarrow \mathrm{A}^{+}+\mathrm{A}^{-}\right)$, through which interacting neutral micelles are converted into charged micelles. The disproportionation model is widely accepted as the charging mechanism for both ionizable and nonionizable surfactant in dielectric liquid [6]. 
When negative and positive charge resulting from dissociation remain confined to the polar core, the reverse micelle remains neutral. But when they collide with each other, they can exchange their contents, resulting in two oppositely charged reverse micelles, and this is called disproportionation $[3,7-9]$.

Reverse micelles have been studied extensively using both experimental and computational techniques. Being a phenomenon that takes place at the molecular level, it is best understood by performing molecular dynamics simulations. Molecular dynamic simulations with full atom model were performed for various surfactant-solvent systems [11-13]. To reduce time required for simulation, coarse grained and united atom models were used for molecular modeling [1425]. To simulate reverse micelles, two distinct approaches can be followed, either by starting from a mixture of nonpolar liquid, surfactant, and water to simulate the self-assembly of molecules to form reverse micelle or else by starting with a preassembled reverse micelle. It has been reported that the simulations performed by following these distinct approaches would yield the same result, which would imply that starting with a preassembled micelle would be advantageous from the point of view of computational cost [26]. Though a real chemical experiment involves a very large number of reverse micelles, to reduce computational time of simulation, usually a single micelle is considered [27]. Most of the simulations have been performed for ionizable surfactant like AOT [11, 13, 20, 21]. Molecular dynamics simulation of formation of reverse micelle in the dodecane/span 80 mixture has not been reported so far, to the best of our knowledge. Except for an experimental work carried out in 1998, little information is available on the formation of reverse micelle in the dodecane/span80 system [28].

Molecular dynamics simulation of movement of reverse micelles under the effect of external electric field is an area which is yet to be properly explored by researchers, and very little information is available in the literature on this topic. A study of mobility, drift, and diffusion of charged OLOA reverse micelles in dodecane is, to our knowledge, the only reported work on the topic [3]. Experimental studies have been conducted to measure the drift velocity of ions and electrons in nonpolar liquids and suggest that though the mobility of ion remains constant at lower electric field, it increases at high electric field [29]. Drift of span 80 reverse micelles in dodecane is a topic which is yet to catch the attention of researchers.

The objective of our study is to perform a molecular dynamics simulation of the dodecane/span 80 system, in order to verify the formation of reverse micelles in the same. In Section 2 of this paper, the molecular model and the method of molecular dynamics simulation are discussed. In Section 3, the presence of charge carriers in dodecane/span 80 mixture by solvation of ions in the core of the reverse micelle is verified by simulation. Section 3 also discusses the movement of reverse micelles under the influence of external electric field, drift speed, critical magnitude of electric field at which ion breaks out of span 80 reverse micelle, and the fraction of charged reverse micelles at varying electric field. Concluding remarks on the work are presented in Section 4.

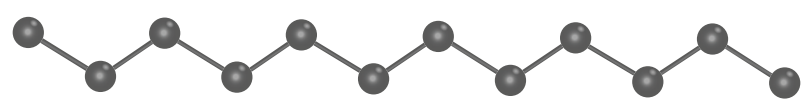

FIGURE 1: United atom model of dodecane, represented by 12 carbon atoms.

\section{Computational Model and Method}

In this work, dodecane was modeled using united atom model, in which 12 united carbon atoms describe the molecule (Figure 1). To model the span80 (sorbitan monooleate) molecule, we used a hybrid model, reported in the literature, which has a hydrophobic tail represented by 17 united carbon atoms and the hydrophilic head represented by an all atom model (Figure 2) [30].

Intermolecular potential for each molecule is calculated by summing bond, angular, and dihedral potentials,

$$
U_{\text {int }}=U_{\text {bond }}+U_{\text {angle }}+U_{\text {dihedral }} \text {. }
$$

Energy due to stretching of bond is calculated by a harmonic potential,

$$
U_{\text {bond }}=K_{b}\left(r-r_{0}\right)^{2},
$$

where $K_{b}$ is bond constant and $r_{0}$ is the equilibrium bond length. Angles are also constrained by a harmonic potential,

$$
U_{\text {bond }}=K_{\theta}\left(\theta-\theta_{0}\right)^{2},
$$

where $K_{\theta}$ is a constant and $\theta_{0}$ is the equilibrium angle. Torsional energy is calculated by using a cosine potential form,

$$
U_{\text {dihedral }}=A[1+\cos (m \phi-\delta)],
$$

where $\phi$ is the dihedral angle.

Nonbonded interactions between atoms are described by Lennard-Jones 12-6 potential and electrostatic potential. Force field parameters for span 80 were taken from previously reported work [31]. $\mathrm{CH}_{3}$ and $\mathrm{CH}_{2}$ groups of the tail portion of span 80 molecule are represented by united atoms in the above model. So the same force field parameters were used for united atoms representing $\mathrm{CH}_{3}$ and $\mathrm{CH}_{2}$ groups of dodecane in this work.

The CHARMM TIP3P model was used for water. Sodium and chloride ions were used to represent impurity ions in the system. For water and ions $\left(\mathrm{Na}^{+}\right.$and $\left.\mathrm{Cl}^{-}\right), \mathrm{CHARMM}$ force field parameters were used [32].

Initial configurations of molecules in the simulation box for each simulation were constructed using the freely distributed Packmol package [33]. All simulations were performed by freeware NAMD [34]. Simulations were conducted on periodic boundary conditions, and long range electrostatics interactions were calculated using the particle mesh Ewald method. Energy minimization was done for $0.5 \mathrm{~ns}$ and all production runs were done using NVT ensemble, with times step of $2 \mathrm{fs}$. Total energy of the system was monitored to determine its equilibrium state. Visualization and various analyses were done using a VMD package [35]. 


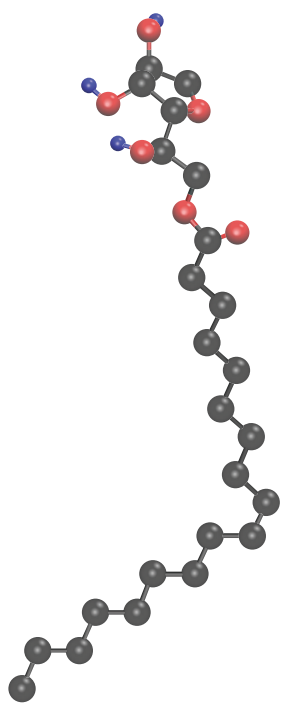

(a)

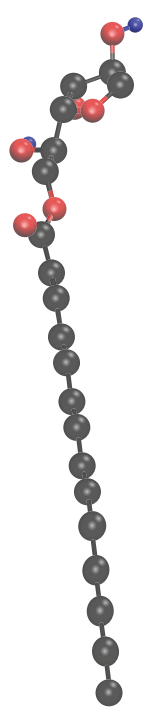

(b)
FIGURE 2: Hybrid model of span 80 molecule viewed from two orthogonal directions, (a) and (b). Carbon atoms are in black, oxygen in red, and hydrogen in blue.

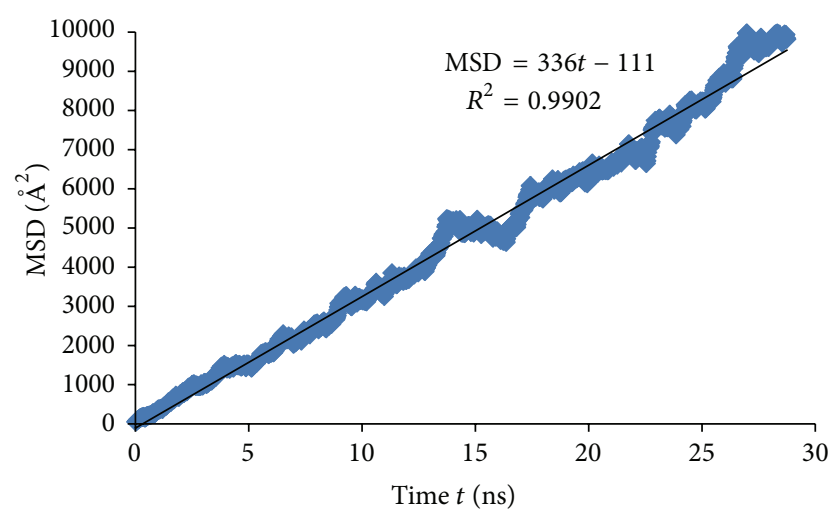

FIGURE 3: MSD-versus-time graph for span80 molecule.

\section{Result and Discussions}

3.1. Validation of Model. Validation of the force field parameters used for simulation is pertinent to make sure that the model is physically meaningful. In this work, the diffusion coefficients of span 80 in dodecane are compared between the results obtained through molecular dynamics simulation and theoretical calculation. To calculate the diffusion coefficient, a simulation box of cubic shape with length of side equal to 44 angstroms consisting of 216 dodecane molecules and one span 80 molecule was made using Packmol. Sizing of the box was corresponding to the macro density of dodecane at $293 \mathrm{~K}$. Simulation was performed for 16 nanoseconds and the root mean squared displacement (RMSD) values for span 80 were extracted using VMD. From the RMSD values, a mean-squared-displacement- (MSD-) versus-time graph was plotted (Figure 3). by

As per Einstein's equation, diffusion coefficient $D$ is given

$$
D=\frac{1}{6} \frac{d(\mathrm{MSD})}{d t} .
$$

MSD-versus-time plot was approximated as a straight line represented by an equation MSD $=336 t-111$, where $t$ is in the unit of ns with the slope being 336. Slope was measured by averaging values obtained over ten different runs of simulations, and the self-diffusion coefficient of span 80 was found to be $5.1 \times 10^{-10} \mathrm{~m}^{2} / \mathrm{s}$.

Since experimental works to calculate diffusion coefficient of span 80 in dodecane could not be found in the literature, we calculated it theoretically. To calculate the diffusion coefficient, we have the Einstein-Stokes equation,

$$
D=\frac{k_{B} T}{6 \pi \eta R},
$$

where $\eta$ is the viscosity of solvent and $R$ is the hydrodynamics radius of the molecule being diffused. From the structure of span80, it can be safely assumed that all atoms are hard spheres in contact with solvent atoms. So, the span 80 molecule can be represented by an equivalent sphere which has a surface area equivalent to the sum of the surface areas of individual atoms of span80. Since we considered a united atom model for the tail part and an all atom model for the head part, to calculate the effective surface area of the molecule, 24 carbon atoms, 6 oxygen atoms, and 3 hydrogen atoms were taken into consideration. Using the values of covalent radius of the above atoms, radius of equivalent sphere was found to be $4.226 \AA$. Substituting the obtained value of the radius, viscosity of the solvent dodecane $\eta$ as $1.34 \times 10^{-3} \mathrm{~Pa} \cdot \mathrm{s}$, and temperature $T$ as $293 \mathrm{~K}$ in (6), we get the diffusion coefficient as $3.789 \times 10^{-10} \mathrm{~m}^{2} / \mathrm{s}$. Since the values of the diffusion coefficient obtained through molecular dynamics simulation and theoretical approximation are of the same order, it can be concluded that the model and the force field parameters used in this work are physically meaningful.

3.2. Formation of Aggregates. To understand the formation of aggregate structure, two different cases of simulation were performed. Number of molecules and size of simulation box in each case are summarized in Table 1. In both cases, concentration of span 80 in the mixture is $5 \%$ by weight. Since computational time is a function of number of atoms, choice of the number of molecules was decided by the available computational resources. Sizing of the box was done according to the macro density of constituents at $293 \mathrm{~K}$ and 1 atmosphere pressure. After minimizing for $0.5 \mathrm{~ns}$, molecular dynamics simulation was performed in NVT ensemble for $40 \mathrm{~ns}$. The trajectory of the molecules in the simulation was visualized using VMD. It was seen that the water molecules form an aggregate and span 80 molecules assemble around it. Different stages of self-assembly for case 1 are shown in Figure 4.

Self-assembly of span 80 molecules around aggregates of water molecules is clear in Figure 4. It can be seen that 


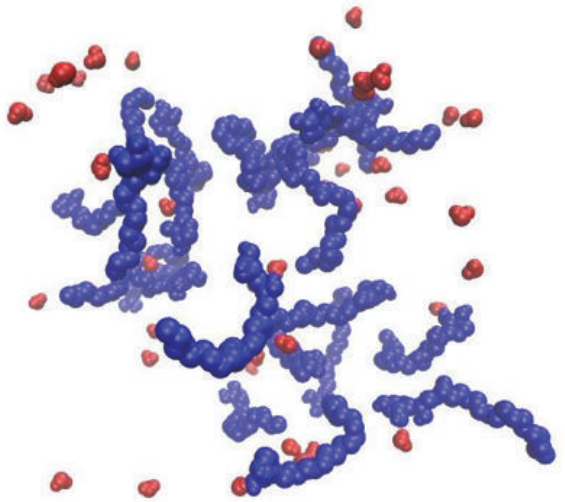

(a)

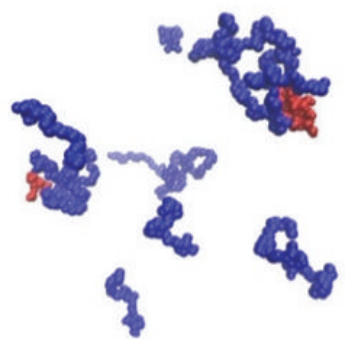

(b)

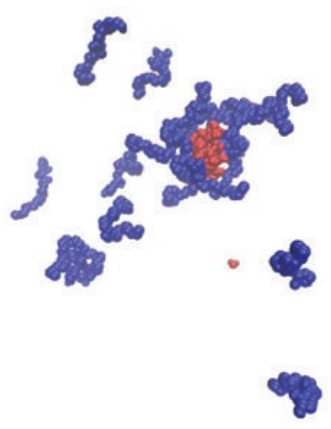

(c)

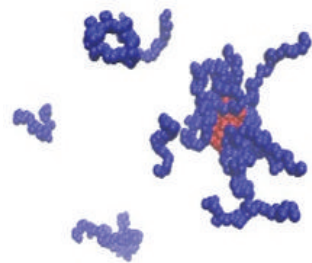

(e)

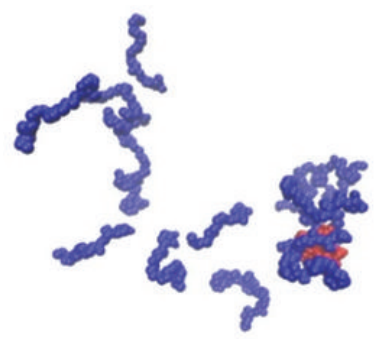

(d)

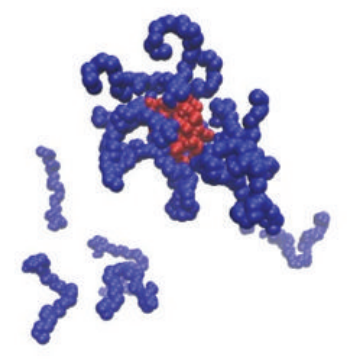

(f)

Figure 4: Configuration at various temporal stages of the case 1 simulation: (a) $t=0 \mathrm{~ns}$, (b) $t=10 \mathrm{ns,} \mathrm{(c)} t=20 \mathrm{~ns}$, (d) $t=30 \mathrm{~ns}$, (e) $t=40 \mathrm{ns,}$ and (f) $t=50$ ns. span 80 molecules are shown in blue and water molecules in red. For clarity, dodecane molecules are not shown.

TABLE 1: Setup for two cases of simulations for formation of aggregates by self-assembly.

\begin{tabular}{lcccc}
\hline $\begin{array}{l}\text { Simulation } \\
\text { case }\end{array}$ & $\begin{array}{c}\text { Number of span80 } \\
\text { molecules }\end{array}$ & $\begin{array}{c}\text { Number of water } \\
\text { molecules }\end{array}$ & $\begin{array}{c}\text { Number of } \\
\text { dodecane molecules }\end{array}$ & Simulation box $(\AA)$ \\
\hline Case 1 & 20 & 40 & 1009 & $76 \times 76 \times 76$ \\
Case 2 & 50 & 100 & 2522 & $102 \times 102 \times 102$ \\
\hline
\end{tabular}

the tail part of span 80 points away from the water core and the head part points towards the water core. This structure corresponds to a reverse micelle. To verify the formation of reverse micelle, two kinds of radial distribution function (RDF) were plotted: the first one between head-group atoms of span 80 and the center of mass of the water core and the second one between the center of mass of the last four united atoms (C29, C30, C31, and C32) representing the tail part of span 80 and the center of mass of the water core (Figure 5(a)). It was found that the first peak in the plot of the RDF between the water core and the head-group is nearer to the water core compared to the first peak in the RDF between the water core and the tail group, which confirms the formation of reverse micelle.

In the second case of simulation, aggregation of water and the span 80 molecules similar to the first case was noticed. 


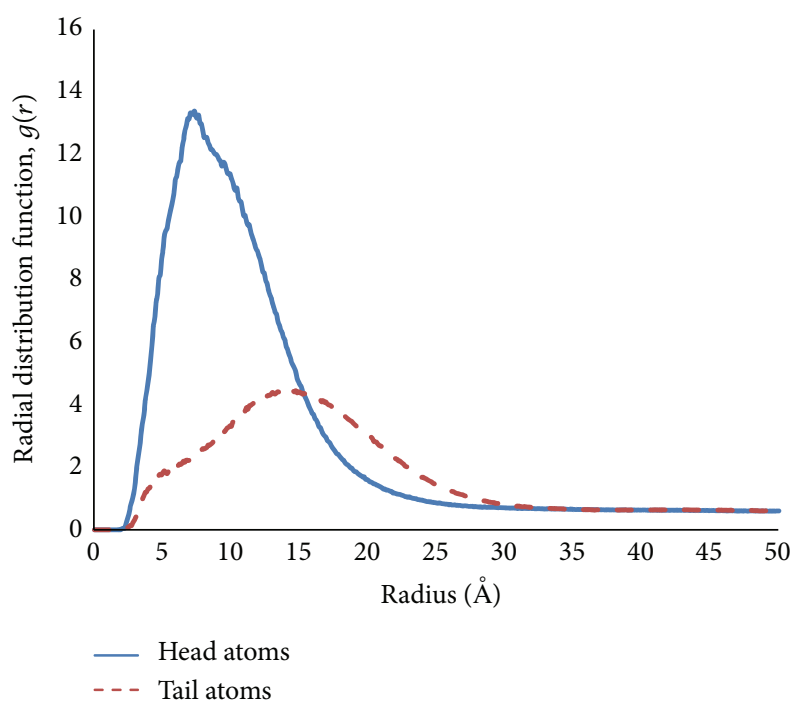

(a)

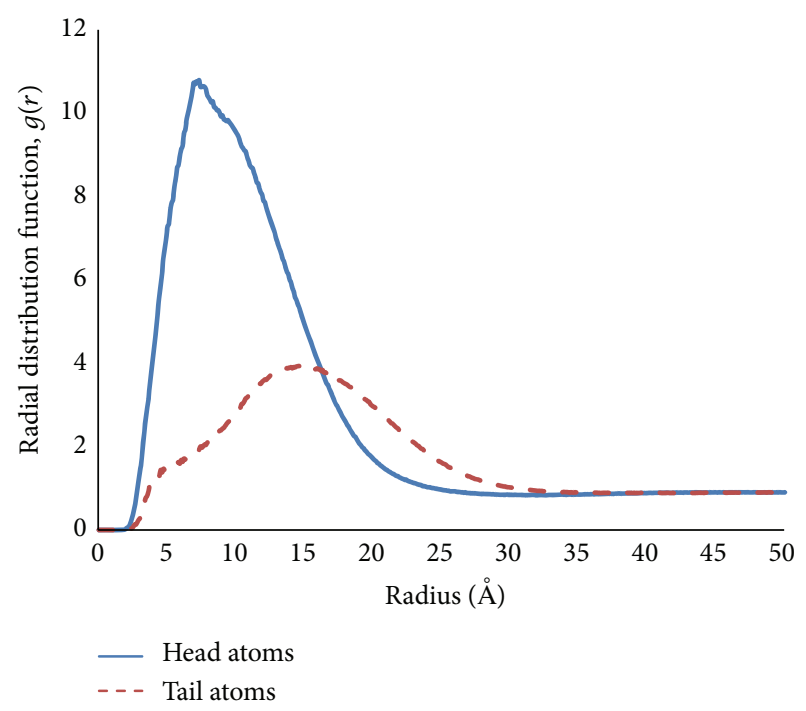

(b)

FIGURE 5: Radial distribution function of head and tail atoms of span80 with water for two cases, (a) case 1 and (b) case 2.

Radial distribution function curves plotted for the second case are given in Figure 5(b). In this case too, the first peak in the plot of the RDF between the water core and the headgroup is nearer to the water core compared to the first peak in $\mathrm{RDF}$ between the water core and the tail group, confirming the formation of reverse micelle.

Approximating the shape of the reverse micelle to be spherical, its radius and the radius of the water core can be estimated from the RDF curves for head and tail group atoms. The peak in RDF between head-group atoms and the water molecules corresponds to the radius of the water core, where head-group atoms are getting attached. Similarly, the peak in $\mathrm{RDF}$ between the center of mass of the last four united atoms (C29, C30, C31, and C32) representing the tail part and the center of mass of the water core corresponds to the radius of the reverse micelle. By averaging the values obtained in 5 different trials of simulation, values for the diameter of reverse micelle and the diameter of water core were found to be approximately $34 \AA$ and $16 \AA$, respectively. The above values are in agreement with the experimental results, which are less than $50 \AA$ and less than $20 \AA$, respectively, for various cases [28].

3.3. Solvation of Ions. Ions are expected to reside inside the water core of reverse micelles. If an equal number of counter ions are present inside the core of a single reverse micelle, then it is a neutral micelle. If the number of counter ions inside the core of a reverse micelle is not the same as that of coions, then the micelle will have a nonzero net charge. Bjerrum length of dodecane is $28 \mathrm{~nm}$, much lower than that of water $(0.7 \mathrm{~nm})$, which means that counter ions in dodecane would recombine very easily. Without reverse micelles, it is impossible for ions to remain free in dielectric liquid.

The same is confirmed through an MD simulation in which the simulation box consists of dodecane, span80, and one sodium and one chloride ion by plotting the distance

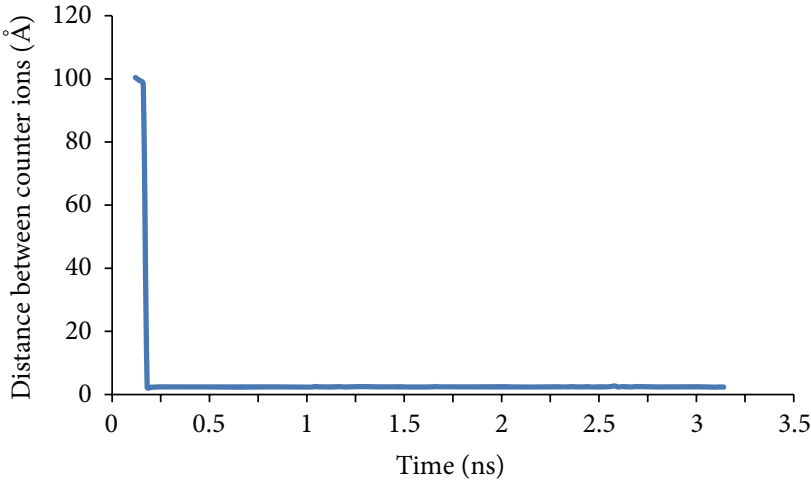

FIgURE 6: Time evolution of the distance between a pair of counter ions in dodecane without water showing the recombination of counter ions near $t=0.2 \mathrm{~ns}$.

between sodium and chloride ions as shown in Figure 6. It can be seen from the graph that, within 0.2 nanoseconds, the distance between counter ions drops to around $2.5 \AA$, which is approximately equal to the bond length of $\mathrm{NaCl}$, from initial separation of $100 \AA$ and remains at the same value. From this example, it can be clearly understood that counter ions cannot exist freely in dielectric liquid.

To study the solvation of ions in reverse micelles, molecular dynamics simulation was performed. Simulation was attempted with random positions of span80, water, sodium, and chloride ions, but it was found to be not feasible since the recombination of counter ions takes place much before the formation of reverse micelles by the process of self-assembly. To overcome this obstacle, simulations were performed with two preassembled reverse micelles, one containing a sodium ion in its core and the other containing chloride atoms. Preassembly was done using Packmol software. Details of preassembled reverse micelles are given in Table 2. Initial 
TABLE 2: Simulation setup for the preassembled reverse micelle to simulate the solvation of ions.

\begin{tabular}{lcccc}
\hline $\begin{array}{l}\text { Number of span80 } \\
\text { molecules }\end{array}$ & $\begin{array}{c}\text { Number of water } \\
\text { molecules }\end{array}$ & $\begin{array}{c}\text { Number of } \\
\text { sodium/chloride ions }\end{array}$ & Diameter $(\AA)$ & Radius of water core $(\AA)$ \\
\hline 40 & 80 & 1 & 34 & 8 \\
\hline
\end{tabular}

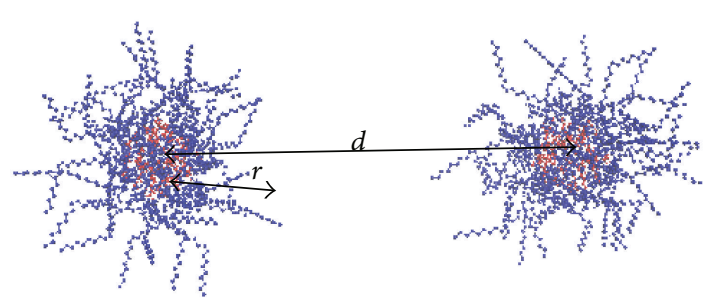

FIGURE 7: Initial configuration of preassembled reverse micelles. span80 molecules are shown in blue and water molecules in red. Sodium ion located at the center of the first micelle and chloride ion at the center of the second micelle are not shown.

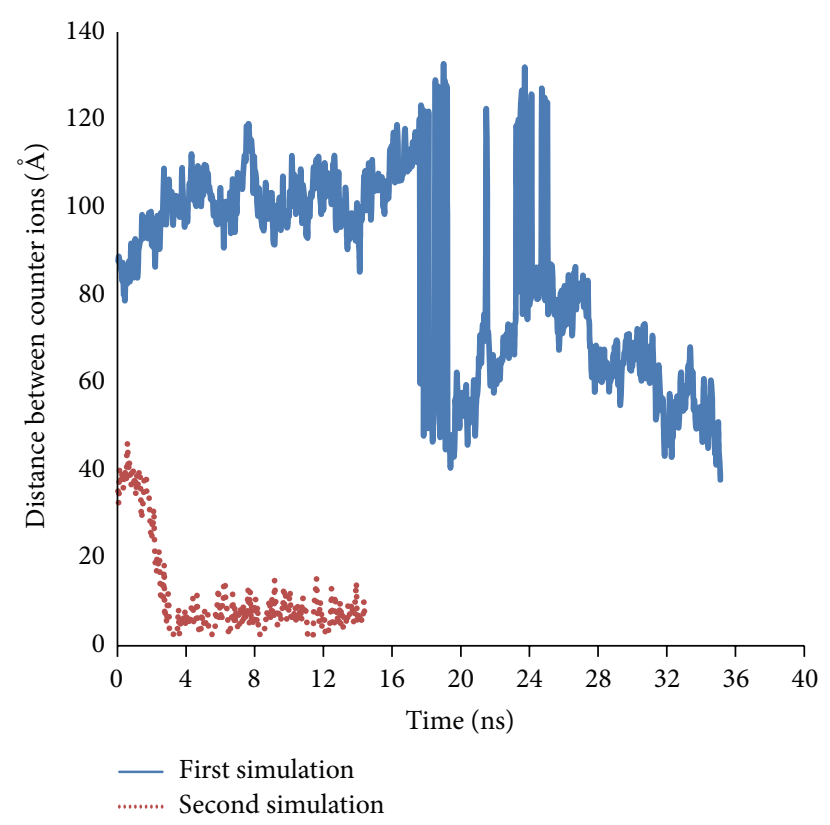

Figure 8: Time evolution of the distance between a pair of counter ions, each being initially included in the core of each of two span 80 reverse micelles in dodecane. In the first simulation, the initial distance between the counter ions is set at almost double that of the second simulation.

configuration of the system for the case of two reverse micelles is shown in Figure 7, where $r$ is the radius of the micelle and $d$ is the distance between the centers of the micelles.

Two simulations were performed in a cubic box of size $152 \AA$ having 8000 dodecane molecules. In each simulation, initial distance between preformed reverse micelles was different. Distance between sodium and chloride ions was plotted and is shown in Figure 8.

In the second simulation it can be seen that counter ions come together to a distance of $2.5 \AA$ after approximately
4 nanoseconds of simulation, which means that they are recombined. Then they dissociate and again recombine, and this cycle continues. The micelles combine together during the process of recombination of ions to form a larger micelle. It was also observed that a few span 80 molecules leave the micelles and exist as monomers in the solution. The process of recombination should result in the reduction of the charge carriers in the solution and obviously lead to the decrease of conductivity.

In the first simulation, counter ions do not recombine and remain solvated in reverse micelles. Since the simulation box size is smaller than the Bjerrum distance of $28 \mathrm{~nm}$, recombination of these ions cannot be ruled out if simulation is extended to a much longer time. Even then, it can be inferred from our results that the counter ions can remain solvated in the water core by the electrostatic shielding provided by reverse micelles.

3.4. Movement under the Effect of External Electric Field. Molecular dynamics simulations of motion of charged reverse micelles were carried out for various magnitudes of electric field. In all cases, electric field was applied along the positive $X$ axis using the inbuilt features of NAMD. One preassembled reverse micelle carrying sodium ion was used for all simulations. The configuration of the preassembled micelle is the same as that given in Table 1 . The number of reverse micelles was restricted to one in the simulations, in order to avoid the Coulomb force between ions, which would affect the drift velocity of reverse micelles.

Theoretically, mobility of ion can be calculated using Stokes equation:

$$
\mu=\frac{e}{6 \pi \eta R}
$$

where $\mu$ is the mobility of ion.

In the present case, radius of reverse micelle is taken as the hydrodynamic radius $(R)$. If mobility is known, drift velocity at a given electric field $E$ can be calculated by the equation

$$
v=\mu E,
$$

where $v$ is the drift velocity and $E$ is the magnitude of the electric field.

To calculate the drift speed of reverse micelles under an external electric field from the trajectory of atoms, coordinates of the sodium ion were saved for each frame. Subsequently, $X$ coordinates were plotted against time and a linear curve was fitted, which showed an acceptable value of regression coefficient. Drift velocity was calculated from the slope of this straight line. Comparison of drift velocity obtained from simulation and that from theoretical calculation for various magnitudes of electric field is given in 


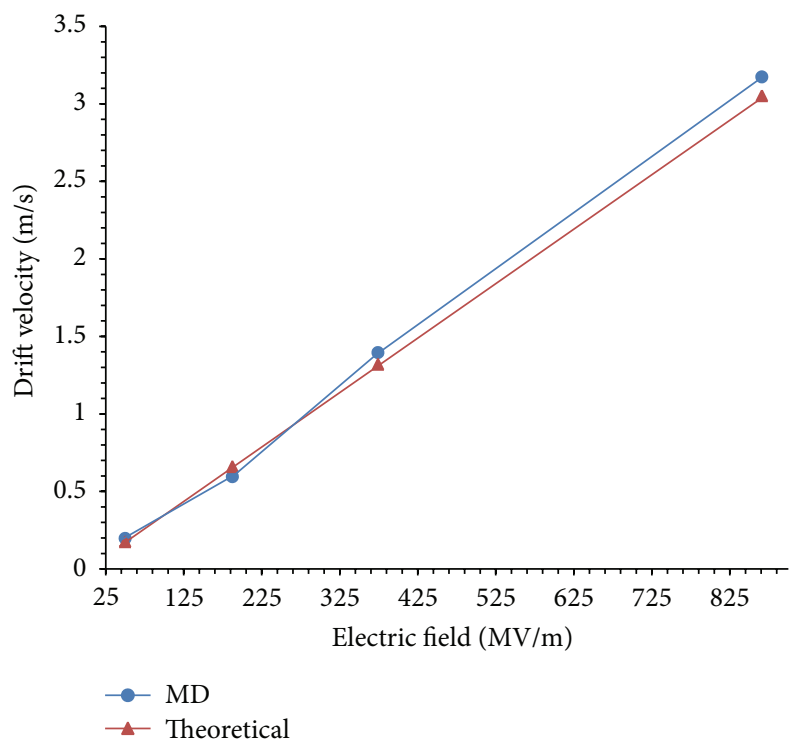

FIGURE 9: Comparison between drift velocity obtained from MD simulation and that from the theoretical calculation.

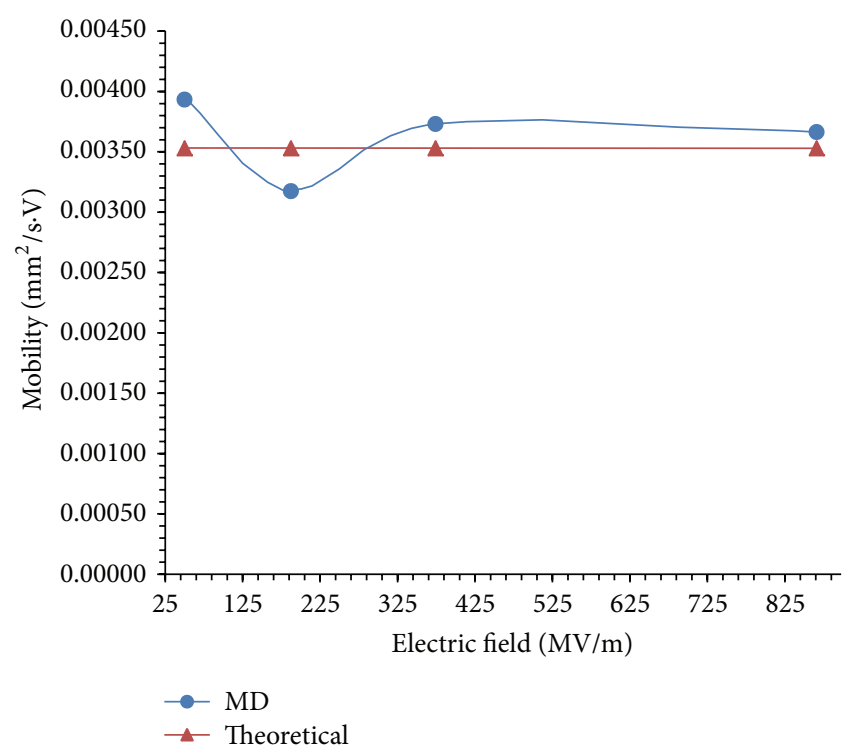

FIGURE 10: Comparison between the ion mobility obtained from MD simulation and that from the theoretical calculation.

Figure 9. It shows excellent agreement between simulation results and theoretical values, and maximum percentage of error is less than 10. Figure 10 shows the comparison of ion mobility values obtained through theoretical calculation and from molecular dynamics simulation. Simulations at lower magnitudes of electric field were also done; however, measureable displacement of reverse micelles was not observed even after a simulation time of $40 \mathrm{~ns}$.

When the applied electric field is very high, reverse micelle becomes unstable and the anion comes out and moves in the direction of the electric field. In our study, we calculated the critical value of the electric field at which the impurity ion inside the core of the span 80 reverse micelle in dodecane

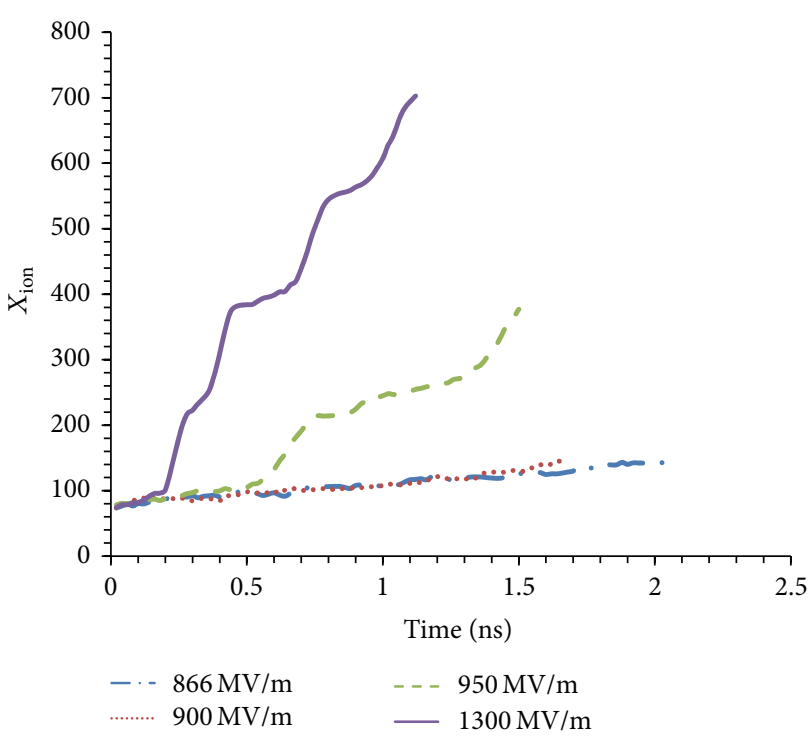

FIGURE 11: Time evolution of the displacement of a cation included initially in a reverse micelle for determination of a critical electric field above which the ion breaks out from the micelle core.

moves out. As in the case of calculating the drift velocity, the $X$ coordinates of the ion $\left(X_{\text {ion }}\right)$ were plotted against simulation time. As long as the ion resides inside the micelle, it moves together with the micelle, and the $X_{\text {ion-versus- }}$ simulation time plot is linear with more or less constant ion mobility. However, when the applied electric field increases, the micelle itself becomes unstable, its water core breaks, and the ion comes out. It moves very fast as a free ion, which is seen as a sharp increase in the slope of the curve (Figure 11). By performing a series of simulations with varying electric field, it was seen that, around a field of $9 \times 10^{8} \mathrm{~V} / \mathrm{m}$, the sodium ion breaks out of the span 80 reverse micelle in dodecane and becomes a free ion.

3.5. Calculation of Fraction of Charged Micelles. Charging of micelles takes place by a disproportionate reaction in which interacting neutral micelles trade their charges to become charged. Large numbers of reverse micelles are expected in the dodecane/span 80 system. Even if a significant number of impurity ions are present in the system, it may not necessarily convert into a high number of charged micelles, since it depends on inter micellar exchanges. So it would be a topic of interest to find out what fraction of the total number of reverse micelles would be charged at a given applied electric field and how this fraction varies with the magnitude of the field.

The number of charged reverse micelles can be found from conductivity by using the equation

$$
\sigma=n z e \mu
$$

where $\sigma$ is the conductivity, $n$ is the number of charged ions per unit volume, and $z$ is the valency of the ion. In this study, only monovalent micelles are considered. The total number of reverse micelles per unit volume can be calculated 


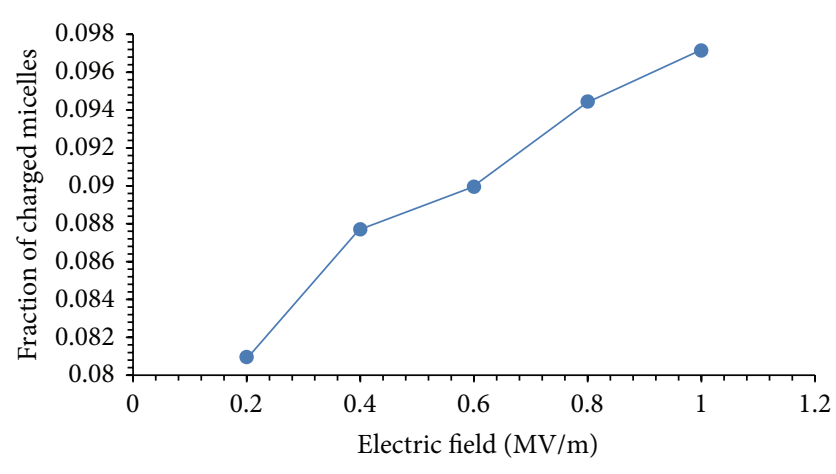

FIGURE 12: Dependence of the ratio of the number of charged reverse micelles to the total number of reverse micelles in dodecane/span 80 mixture on the applied electric field obtained from experimental data on electrical conductivity presented by Suh and Baek [10].

by assuming that all span 80 molecules form reverse micelles with an aggregated number of 40. Experimental values for conductivity at varying magnitude of electric field were taken from previously reported work by Suh and Baek [10]. Variation of fraction of charged micelles with electric field is shown in Figure 12. The fraction of charged micelles is quite small and is found to be increasing with increase in the magnitude of applied electric field. This is understandable, since conductivity increases with increase in the applied electric filed.

\section{Conclusion}

As envisaged in this study, formation of reverse micelles in a dodecane/span80 system was confirmed through molecular dynamics simulation. Physical dimensions of the reverse micelles are in good agreement with the available experimental results in the literature. The fact that impurity ions can remain solvated in the core of reverse micelles was also clearly seen in our simulations. Drift velocity of the reverse micelle under the effect of the external field was also calculated through simulation and it is consistent with the theoretical calculation. The critical magnitude of electric field at which the ion inside the water core goes out of the reverse micelle was calculated by performing a series of simulations. From the available conductivity data, the fraction of charged micelles in a unit volume of the dodecane/span80 system was also calculated and the direct relationship between this fraction and the magnitude of the applied electric field was also shown. It can be concluded that our work explains the physics behind the increase in conductivity of dodecane with the addition of span 80 molecules. The disproportionate reaction through which neutral reverse micelles interchange their charges to become electrically charged is a phenomenon of significant interest and relevance. However, molecular dynamics simulation of the same would require much larger computational resources and time. In our opinion, it is critical for researchers in this field to undertake such a study, which would help us to understand the charging mechanism of nonpolar liquids with much more clarity.

\section{Conflict of Interests}

The authors declare that there is no conflict of interests regarding the publication of this paper.

\section{Acknowledgments}

This work was supported by NRF Grant no. 2009-0083510 through Multi-Phenomena CFD Engineering Research Center and by the Human Resources Development of the Korea Institute of Energy Technology Evaluation and Planning (KETEP) grant funded by the Korean Government Ministry of Knowledge Economy (no. 20114030200030).

\section{References}

[1] Y. K. Suh, "Modelling and simulation of ion transport in dielectric liquids-fundamentals and review," IEEE Transactions on Dielectrics and Electrical Insulation, vol. 19, no. 3, pp. 831-848, 2012.

[2] I. D. Morrison, "Electrical charges in nonaqueous media," Colloids and Surfaces A: Physicochemical and Engineering Aspects, vol. 71, no. 1, pp. 1-37, 1993.

[3] F. Beunis, F. Strubbe, M. Karvar, O. Drobchak, T. Brans, and K. Neyts, "Inverse micelles as charge carriers in nonpolar liquids: characterization with current measurements," Current Opinion in Colloid and Interface Science, vol. 18, no. 2, pp. 129-136, 2013.

[4] A. S. Dukhin and P. J. Goetz, "How non-ionic 'electrically neutral' surfactants enhance electrical conductivity and ion stability in non-polar liquids," Journal of Electroanalytical Chemistry, vol. 588, no. 1, pp. 44-50, 2006.

[5] A. Dukhin, "Critical concentration of ion-pairs formation in nonpolar media," Electrophoresis, vol. 35, no. 12-13, pp. 17731781, 2014.

[6] Q. Guo, V. Singh, and S. H. Behrens, "Electric charging in nonpolar liquids because of nonionizable surfactants," Langmuir, vol. 26, no. 5, pp. 3203-3207, 2010.

[7] F. Strubbe, A. R. M. Verschueren, L. J. M. Schlangen, F. Beunis, and K. Neyts, "Generation current of charged micelles in nonaqueous liquids: measurements and simulations," Journal of Colloid and Interface Science, vol. 300, no. 1, pp. 396-403, 2006.

[8] G. S. Roberts, R. Sanchez, R. Kemp, T. Wood, and P. Bartlett, "Electrostatic charging of nonpolar colloids by reverse micelles," Langmuir, vol. 24, no. 13, pp. 6530-6541, 2008.

[9] A. Molski and E. Dutkiewicz, "Conductivity of water-in-oil microemulsions; Fluctuations from the charge generationrecombination equilibrium," Colloid \& Polymer Science, vol. 271, no. 12, pp. 1177-1182, 1993.

[10] Y. K. Suh and K. H. Baek, "Competition between the bulk and the dissociation layer in electrohydrodynamic flow of dielectric liquid around coplanar electrodes," Physical Review E, vol. 87, no. 2, Article ID 023009, 2013.

[11] A. V. Nevidimov and V. F. Razumov, "Molecular dynamics simulations of AOT reverse micelles' self-assembly," Molecular Physics, vol. 107, no. 20, pp. 2169-2180, 2009.

[12] J.-B. Maillet, V. Lachet, and P. V. Coveney, "Large scale molecular dynamics simulation of self-assembly processes in short and long chain cationic surfactants," Physical Chemistry Chemical Physics, vol. 1, no. 23, pp. 5277-5290, 1999.

[13] S. Abel, F. Sterpone, S. Bandyopadhyay, and M. Marchi, "Molecular modeling and simulations of AOT-water reverse micelles 
in isooctane: structural and dynamic properties," Journal of Physical Chemistry B, vol. 108, no. 50, pp. 19458-19466, 2004.

[14] R. Allen, S. Bandyopadhyay, and M. L. Klein, "C12E2 reverse micelle: a molecular dynamics study," Langmuir, vol. 16, no. 26, pp. 10574-10552, 2000.

[15] S. A. Sanders and A. Z. Panagiotopoulos, "Micellization behavior of coarse grained surfactant models," Journal of Chemical Physics, vol. 132, no. 11, Article ID 114902, 2010.

[16] D. Brown and J. H. R. Clarke, "Molecular dynamics simulation of a model reverse micelle," Journal of Physical Chemistry, vol. 92, no. 10, pp. 2881-2888, 1988.

[17] R. Wu, M. Deng, B. Kong, and X. Yang, "Coarse-grained molecular dynamics simulation of ammonium surfactant selfassemblies: micelles and vesicles," The Journal of Physical Chemistry B, vol. 113, no. 45, pp. 15010-15016, 2009.

[18] A. V. Sangwai and R. Sureshkumar, "Coarse-grained molecular dynamics simulations of the sphere to rod transition in surfactant micelles," Langmuir, vol. 27, no. 11, pp. 6628-6638, 2011.

[19] S. V. Burov, N. P. Obrezkov, A. A. Vanin, and E. M. Piotrovskaya, "Molecular dynamic simulation of micellar solutions: a coarsegrain model," Colloid Journal, vol. 70, no. 1, pp. 1-5, 2008.

[20] E. N. Brodskaya and G. V. Mudzhikova, "Molecular dynamics simulation of AOT reverse micelles," Molecular Physics, vol. 104, no. 22-24, pp. 3635-3643, 2006.

[21] G. V. Mudzhikova and E. N. Brodskaya, "Molecular simulation of an aerosol OT reverse micelle: 1 . The shape and structure of a micelle," Colloid Journal, vol. 68, no. 6, pp. 729-737, 2006.

[22] W. Shinoda, R. DeVane, and M. L. Klein, "Coarse-grained molecular modeling of non-ionic surfactant self-assembly," Soft Matter, vol. 4, no. 12, pp. 2454-2462, 2008.

[23] B. G. Levine, D. N. LeBard, R. Devane, W. Shinoda, A. Kohlmeyer, and M. L. Klein, "Micellization studied by GPUaccelerated coarse-grained molecular dynamics," Journal of Chemical Theory and Computation, vol. 7, no. 12, pp. 4135-4145, 2011.

[24] W. Ding, S. Lin, J. Lin, and L. Zhang, "Effect of chain conformational change on micelle structures: experimental studies and molecular dynamics simulations," The Journal of Physical Chemistry B, vol. 112, no. 3, pp. 776-783, 2008.

[25] S. Abel, M. Waks, M. Marchi, and W. Urbach, "Effect of surfactant conformation on the structures of small size nonionic reverse micelles: a molecular dynamics simulation study," Langmuir, vol. 22, no. 22, pp. 9112-9120, 2006.

[26] A. V. Nevidimov and V. F. Razumov, "Molecular dynamics simulation of reverse micelles: a search for the most efficient strategy," Colloid Journal, vol. 75, no. 2, pp. 191-197, 2013.

[27] A. V. Nevidimov, "Molecular dynamics simulation of reverse micelles: standing problems after 25 years of research," Russian Journal of Physical Chemistry B, vol. 8, no. 4, pp. 554-558, 2014.

[28] I. Abou-Nemeh and H. J. Bart, "Microstructures in the system water/D2EHPA/Span-80/n-dodecane," Langmuir, vol. 14, no. 16, pp. 4451-4459, 1998.

[29] W. F. Schmidt, G. Bakale, A. Khrapak, and K. Yoshino, "Drift velocity of ions and electrons in nonpolar dielectric liquids at high electric field strength," in Proceedings of the IEEE International Conference on Dielectric Liquids (ICDL '11), Trondheim, Norway, June 2011.

[30] S. Han, "Molecular dynamics simulation of sorbitan monooleate bilayers," Bulletin of the Korean Chemical Society, vol. 34, no. 3, pp. 946-948, 2013.
[31] E. NunezRojas and H. Dominguez, "Aggregate structures of the sorbitan monooleate (SPAN80) surfactant at $\mathrm{TiO}_{2}$ (rutile)/water interfaces by computer simulations," Revista Mexicana de Física E, vol. 59, pp. 530-539, 2013.

[32] CHARMM, Chemistry at Harvard Macromolecular Mechanics, http://www.charmm.org/old_site/.

[33] L. Martinez, R. Andrade, E. G. Birgin, and J. M. Martínez, "PACKMOL: a package for building initial configurations for molecular dynamics simulations," Journal of Computational Chemistry, vol. 30, no. 13, pp. 2157-2164, 2009.

[34] J. C. Phillips, R. Braun, W. Wang et al., "Scalable molecular dynamics with NAMD," Journal of Computational Chemistry, vol. 26, no. 16, pp. 1781-1802, 2005.

[35] W. Humphrey, A. Dalke, and K. Schulten, "VMD: visual molecular dynamics," Journal of Molecular Graphics, vol. 14, no. 1, pp. 33-38, 1996. 

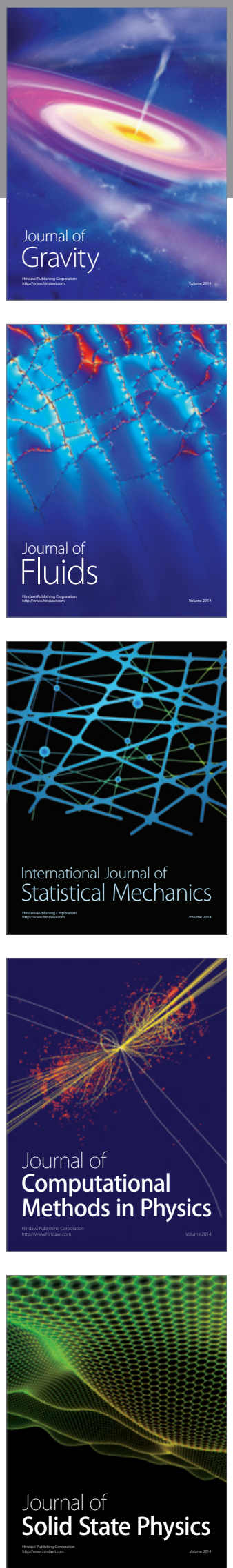

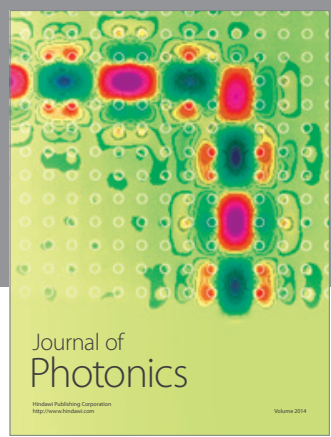

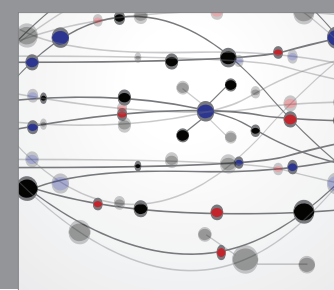

The Scientific World Journal

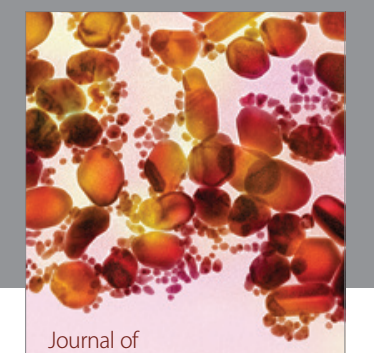

Soft Matter
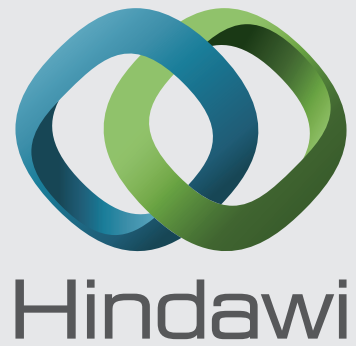

Submit your manuscripts at

http://www.hindawi.com
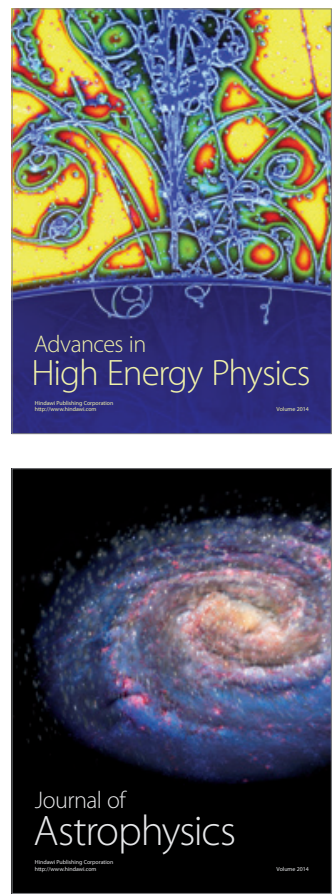
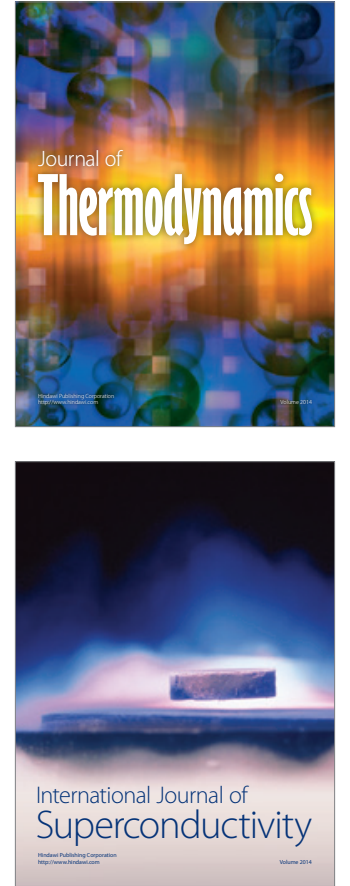
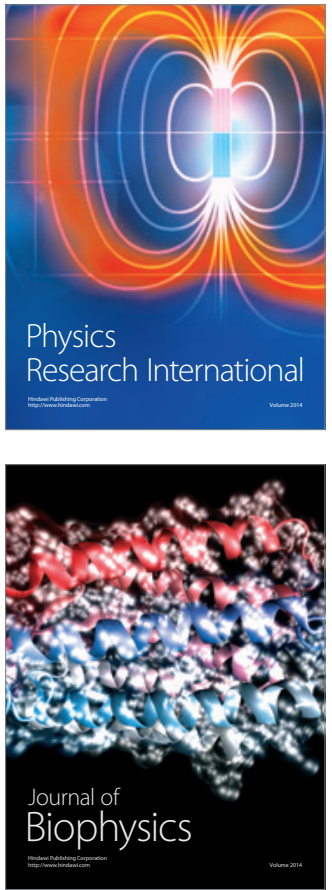
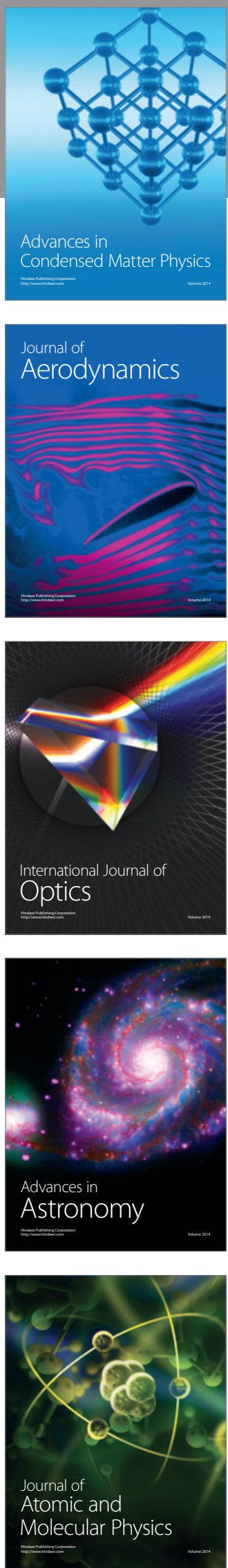